\title{
Tailored hybrid hyperbranched polyglycidol-silica nanocomposites with high third-order nonlinearity
}

\author{
Irina Postnova ${ }^{1}$, Alexander Bezverbny ${ }^{2}$, Sergey Golik², Yury Kulchin² ${ }^{2}$ Haiqing Li ${ }^{3}$, Jing Wang ${ }^{3}$, II Kim³ , \\ Chang-Sik $\mathrm{Ha}^{3}$ and Yury Shchipunov $3,4^{*}$
}

\begin{abstract}
One of the most convenient techniques for optical material fabrication is the sol-gel processing. It can be performed at low temperature that enables one to entrap even relatively unstable organic substances into silica matrix at the nanometer scale, thus developing homogeneous hybrid organic-inorganic nanocomposite materials of various functionalities. Here, novel hybrid organic-inorganic nanocomposites with good optical transparency and high third-order nonlinearity were prepared biomimetically through the mineralization of dendritic macromolecules (hyperbranched polyglycidols) using a compatible ethylene glycol-containing silica precursor. The synthesis was performed at neutral pH media in aqueous solutions without addition of organic solvents at ambient conditions owing to the catalysis of processing. Polyglycidols provided also the formation of gold nanoparticles localized in their core. They served as reducing and stabilizing agents. It is shown that trace amounts of nanoparticles could regulate nonlinear properties of a nanocomposite. High nonlinearity manifests itself in a supercontinuum generation at remarkably short lengths $c a .1 \mathrm{~mm}$. The phenomenon consists of filamentous intense white lighting due to the spectral broadening of initial ultrashort (femtosecond) laser pulses propagating through the material. The developed hybrid nanocomposites possessing large nonlinearity, high-speed optical response, stability under intense lighting, lowcost, and easy preparation are promising for a diverse range of applications as active components for all-optical signal processing from chemical sensing to biological cell imaging and lighting control in telecommunication.
\end{abstract}

Keywords: Hyperbranched polyglycidol, Gold nanoparticles, Sol-gel, Organic-inorganic nanocomposite, Third-order nonlinearity, Photonic material, Supercontinuum generation

\section{Findings}

\section{Introduction}

Mineralized tissues of living organisms reveal properties that material scientists can only aspire to achieve. Basalia spicules of glass sponges provide such an example. They have unique optical properties that are controlled through sophisticated structural organization of biosilica [1-3]. In particular, their optical nonlinearity is higher than that of quartz fibers [4]. We also showed in this work that the hybrid polysaccharide-silica nanocomposites prepared by

\footnotetext{
* Correspondence: yury.shchipunov@googlemail.com

${ }^{3}$ The WCU Center for Synthetic Polymer Bioconjugate Hybrid Materials, Department of Polymer Science and Engineering, Pusan National University, San 30, Jangjeon-dong, Geumjeong-gu, Busan 609-735, South Korea ${ }^{4}$ Institute of Chemistry, Far East Department, Russian Academy of Sciences, pr. 100 let Vladivostoku 159, Vladivostok 690022, Russia

Full list of author information is available at the end of the article
}

biomimicking mineralization demonstrated similar high third-order nonlinear susceptibility. Here, we use hyperbranched polyglycidols (HBPs) instead of polysaccharides to synthesize novel photonic materials.

\section{Results and discussion}

HBPs (see structural formula in Figure 1a) fall into dendritic macromolecules with random branch-onbranch topology [5]. They were synthesized and characterized as detailed in our article [6]. Macromolecules have a spherical or elliptical shape with rather densely packed chains. A scanning electron microscope (SEM) image can be seen in Figure 1d.

HBPs have aliphatic polyether backbones containing multiple terminal hydroxyl groups like polysaccharides. This enabled us to apply the same biomimicking mineralization 

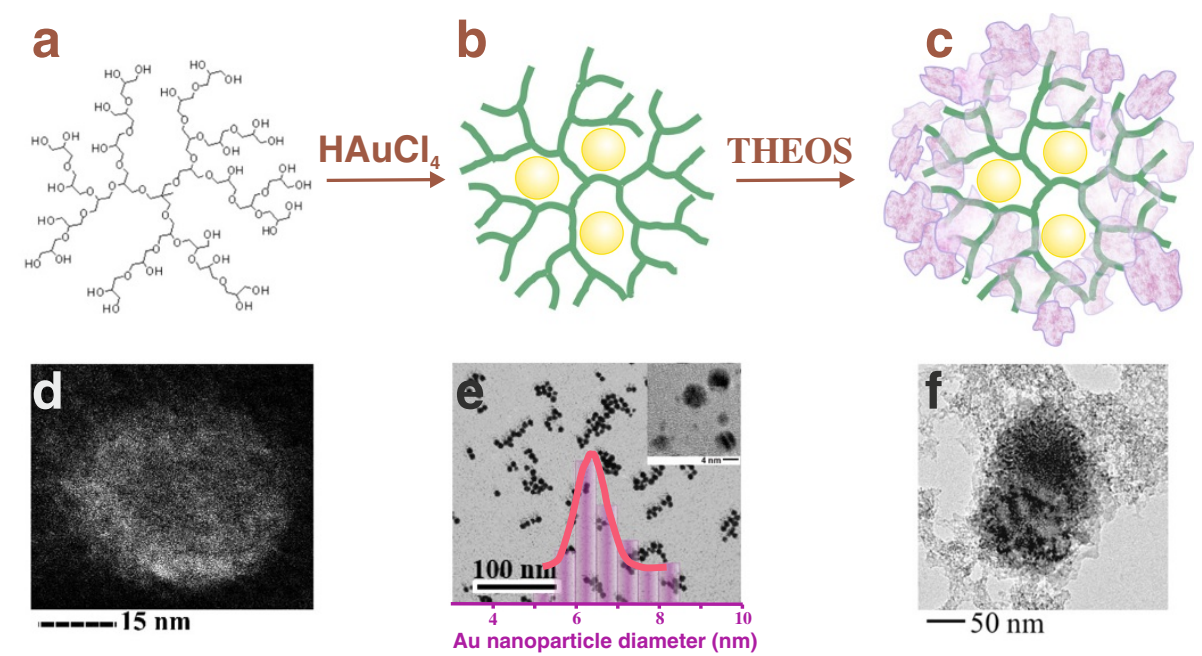

Figure 1 Schematic presentation of main stages of preparation of hybrid HBP-silica nanocomposites with entrapped gold nanoparticles. (a) Structural formula and (d) high-resolution SEM image of the HBP molecule; (b) schematic drawing of the HBP molecule with gold nanoparticles in its core, (e) TEM image of gold nanoparticles and diagram of their size distribution; (c) schematic presentation and (f) TEM image of the mineralized HBP molecule.

procedure by treating them with a silica precursor, tetrakis (2-hydroxyethyl)orthosilicate (THEOS). Its advantage is its excellent compatibility with biopolymers and HBPs. Syntheses are performed in one stage at neutral $\mathrm{pH}$, without the addition of acid/alkali and heating because of the catalysis of hydrolysis by hydroxyl-containing substances $[7,8]$. After a time of admixing an appropriate amount of THEOS into an aqueous solution of HBP, one can find silica formation that is manifested in the solution jellification. A representative transmission electron microscope (TEM) image of the synthesized nanocomposite is presented in Figure 1f. There are HBP macromolecules encased in the silica matrix.

HBPs were used to synthesize gold nanoparticles as reported first in $[6,9]$ by adding a chloroauric acid into a HBP solution. They serve as both reducing and stabilizing agents. Gold nanoparticles thus formed are mainly located within, rather than the outside of, the HPB core. As seen in Figure 1e and the inserted size distribution diagram, their diameter is around $5 \mathrm{~nm}$.

As-synthesized and dried nanocomposites were optically transparent. The transparency depended on the silica concentration. Samples synthesized using 10 wt.\% THEOS have slight opalescence. With the increasing silica concentration, the transmittance is improving (curve 1, Figure 2a). It is related to the morphology of mineralized HBPs. Representative SEM images are given in Figure 2b,c. As obvious, nanocomposites prepared with $10 \mathrm{wt} . \%$ THEOS are rather heterogeneous, consisting of micron- and submicron-sized clusters (Figure $2 \mathrm{~b}$ ). Their presence could be a reason of the mentioned opalescence. The sample containing $50 \mathrm{wt} . \%$ silica is much homogeneous, consisting of smaller clusters (Figure 2c). This is the reason for improving the transparency with the increasing THEOS concentration.
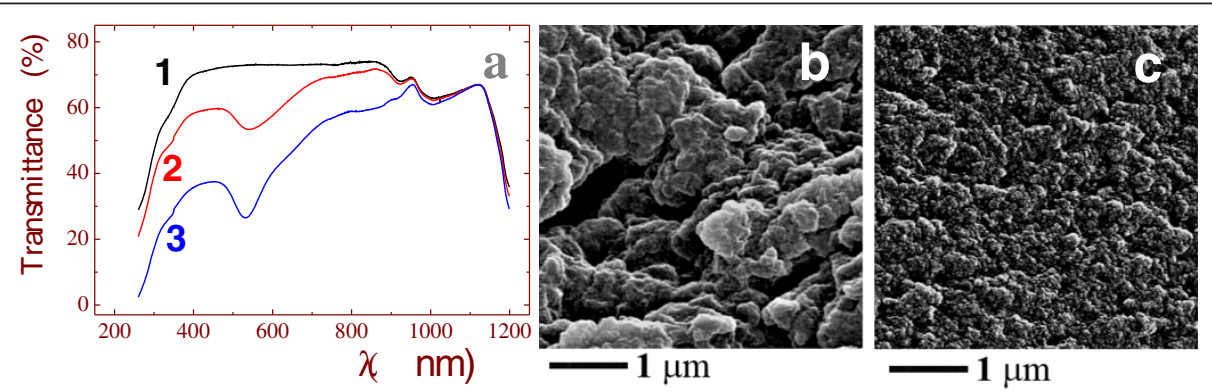

Figure $\mathbf{2}$ Characterization of transmittance (a) and morphology (b, c) of hybrid nanocomposites. (a) Transmittance of HBP-silica nanocomposites synthesized in an aqueous solution containing 50 wt.\% THEOS and 1 wt.\% HBP (1) with addition of $2.0 \times 10^{-5}$ (2) and $3.8 \times 10^{-5} \mathrm{M}$ $\mathrm{HAuCl}_{4}$ (3). (b, c) SEM images of HBP-silica nanocomposites. The THEOS concentration was 10 (b) and 50 wt.\% (c); HBP, 1 (b) and 5 wt.\% (c). Syntheses were performed at ambient conditions. 
The transmittance is very sensitive to the presence of even trace amount of gold nanoparticles (curves 2 and 3, Figure 2a). The effect is caused by the Au surface plasmon resonance that follows from a minimum located at a wavelength of $528 \mathrm{~nm}$ for the sample prepared at $3.8 \times 10^{-4} \mathrm{M}$ chloroauric acid (curve 3, Figure 2a).

The optical nonlinearity of the developed hybrid HBPsilica nanocomposites was examined by means of the standard Z-scan technique $[4,10]$. As found, the nonlinear refractive index $n_{2}$ is equal to $2.5 \times 10^{-13} \mathrm{~cm}^{2} / \mathrm{W}$ at $\lambda=532 \mathrm{~nm}$ and a pulse duration of $5 \mathrm{~ns}$. For reference, the $n_{2}$ value of fused silica, which is widely used as nonlinear photonic fibers, is $2.6 \times 10^{-16} \mathrm{~cm}^{2} / \mathrm{W}$ (Appendix $B$ in [11]). One may see from the comparison that the nonlinearity of hybrid HBP-silica nanocomposites is three orders of magnitude greater.

One of the impressive manifestations of the high nonlinearity of developed hybrid HBP-silica nanocomposites is the supercontinuum generation. Its appearance is obvious only under the action of ultrashort laser pulses. Their duration in our experiments was 40 fs with a frequency of $100 \mathrm{~Hz}$ and a central wavelength of $800 \mathrm{~nm}$. A schematic drawing of the experimental setup is presented in Figure 3a. More details are given in our article [4]. When an unfocused laser beam of $7 \mathrm{~mm}$ in diameter with maximum energy of a pulse of $1 \mathrm{~mJ}$ comes through a sample, an appearance of multiple distinct filaments is observed in its bulk (Figure 3b). As obvious in Figure 3c, an outgoing light beam constitutes a bright white core surrounded by spectral colors.

Spectral characteristics of the supercontinuum can be seen in Figure 3d. Curve 1 presents the initial spectrum of the laser beam. Emitted spectrum for the sample containing HBP is shown by curve 2; for samples with Au nanoparticles of various concentrations, by curves 3 and 4 . The nanosized gold serves as a dopant, having a profound effect on the supercontinuum generation. The light intensity measured in the sample with their trace amounts (curve 3) is around an order of magnitude higher than that in the HBP-silica nanocomposite (curve 2) almost over the whole visible spectral region (420 to $720 \mathrm{~nm}$ ). As followed from a comparison of curves 3 and 4 in Figure 3d, the effect depends on the Au nanoparticle content. When a critical concentration is reached, their successive addition causes a sharp decay of emission. The bleaching means that the plasmon resonance of gold nanoparticles has a profound effect on the processes in the bulk material.

The conversion of ultrafast laser pulses in the supercontinuum spectrum occurs at remarkably short lengths. We observe it in hybrid HBP-silica nanocomposites doped with $\mathrm{Au}$ nanoparticles with a thickness of $0.9 \mathrm{~mm}$. It is essential that hybrids are stable under the action of intense laser pulses. Notable changes in the optical properties are

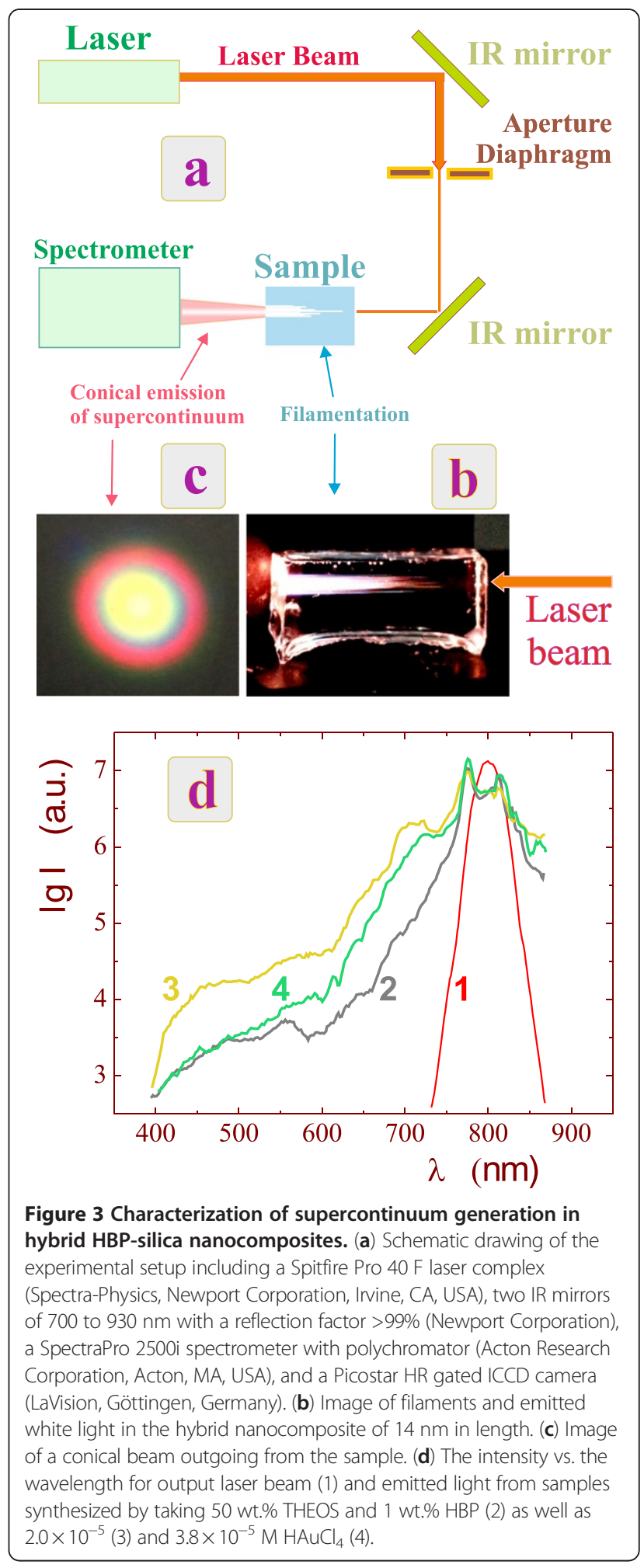

not mentioned after prolonged (hours) testing. The value of nonlinear refractive index is indicative of possible ultrafast processes responsible for the supercontinuum generation. When its value is around $10^{-16}$ or $10^{-13} \mathrm{~cm}^{2} / \mathrm{W}$, the phenomenon is brought about by electronic polarization 
or molecular orientation and electrostriction, respectively, which is characterized by ultrashort response time $\left(10^{-15}\right.$, $10^{-12}$, and $10^{-9}$, respectively; see, e.g., [12]). The measured value of $2.5 \times 10^{-13} \mathrm{~cm}^{2} / \mathrm{W}$ at a pulse duration of $5 \mathrm{~ns}$ and the supercontinuum emission at laser pulses of 40 fs mean that the phenomenon is generated jointly by a number of ultrafast processes.

\section{Conclusions}

This study extends a number of novel biomimetic organicinorganic hybrids possessing high nonlinear susceptibility that was found previously for polysaccharide-silica nanocomposites in [4]. Here, we demonstrated additionally that the optical nonlinearity can be regulated by trace amounts of gold nanoparticles. As dopants, nanosized noble metal, metal oxides, and quantum dots are applied rather widely for regulating the photonic properties of glasses and sol-gel-derived silicates (see, e.g., [13-15]) but not, to our knowledge, for enhancing the supercontinuum processing. We have developed a novel type of photonic nanocomposite materials consisting of mineralized dendritic and polysaccharide macromolecules of which the structure is tailored via biomimetic mineralization with the help of a compatible precursor and the entrapment of gold nanoparticles. Their advantage is in the easy processability because of low viscosity of the initial solution of HBP, its low-cost synthesis, intrinsic transparency, and excellent nonlinear optical properties. They can be integrated with various materials. Owing to the high nonlinear refractive index, the supercontinuum is generated at short lengths. This is very promising for such potential applications as planar optical devices for optical computers, optical correlators, femtosecond Kerr shutter, and sensoring.

\section{Abbreviations}

HBPs, hyperbranched polyglycidols; THEOS, tetrakis(2-hydroxyethyl) orthosilicate; SEM, scanning electron microscope.

\section{Competing interests}

The authors declare that they have no competing interests.

\section{Authors' contributions}

IP prepared and examined the properties of hybrid nanocomposites, carried out the physico-chemical studies, acquired and analyzed the data, and helped draft the manuscript. AB conceived the photonic study, acquired and analyzed the data, helped draft the manuscript, and gave a final approval of the version for publication. SG carried out the photonic studies and acquired and analyzed the data. YK conceived the study and helped draft the manuscript. HL synthesized, purified, and analyzed the HBPs and acquired and analyzed the data. JW synthesized, purified, and analyzed the HBPs and acquired the data. IK designed the HBP and gold nanoparticle synthesis strategy, analyzed the data, and drafted the manuscript. CSH conceived the study and gave a final approval of the version for publication. YS conceived, designed, and coordinated the study, analyzed the data, and prepared and submitted the final version for publication. All authors read and approved the final manuscript.

\section{Author details}

${ }^{1}$ Chemical Department, Far East Federal University, ul. Sukhanova 8, Vladivosotk 690050, Russia. ${ }^{2}$ Institute of Automation and Control Processes, Far East Department, Russian Academy of Sciences, ul. Radio 5, Vladivostok
690041, Russia. ${ }^{3}$ The WCU Center for Synthetic Polymer Bioconjugate Hybrid Materials, Department of Polymer Science and Engineering, Pusan National University, San 30, Jangjeon-dong, Geumjeong-gu, Busan 609-735, South Korea. ${ }^{4}$ Institute of Chemistry, Far East Department, Russian Academy of Sciences, pr. 100 let Vladivostoku 159, Vladivostok 690022, Russia.

Received: 20 May 2012 Accepted: 20 June 2012

Published: 12 July 2012

\section{References}

1. Aizenberg, J., Sundar, V.C., Yablon, A.D., Weaver, J.C., Chen, G.: Biological glass fibers: correlation between optical and structural properties. Proc. Nat. Acad. Sci. USA 101, 3358-3363 (2004)

2. Schroder, H.C., Brandt, D., Schlossmacher, U., Wang, X.H., Tahir, M.N., Tremel, W., Belikov, S.I., Muller, W.E.G.: Enzymatic production of biosilica glass using enzymes from sponges: basic aspects and application in nanobiotechnology (material sciences and medicine). Naturwissenschaften 94, 339-359 (2007)

3. Schroder, H.C., Wang, X.H., Tremel, W., Ushijima, H., Muller, W.E.G.: Biofabrication of biosilica-glass by living organisms. Nat. Prod. Rep. 25 455-474 (2008)

4. Kulchin, Y.N., Bezverbny, A.V., Bukin, O.A., Voznesensky, S.S., Golik, S.S., Mayor, A.Y., Shchipunov, Y.A., Nagorny, I.G.: Nonlinear optical properties of biomineral and biomimetical nanocomposite structures. Laser Phys. 21, 630-636 (2011)

5. Wilms, D., Stiriba, S.E., Frey, H.: Hyperbranched polyglycerols: from the controlled synthesis of biocompatible polyether polyols to multipurpose applications. Accounts Chem. Res. 43, 129-141 (2009)

6. Li, H., Jo, J.K., Zhang, L.D., Ha, C.S., Suh, H., Kim, I.: Hyperbranched polyglycidol assisted green synthetic protocols for the preparation of multifunctional metal nanoparticles. Langmuir 26, 18442-18453 (2010)

7. Shchipunov, Y.A., Karpenko, T.Y.: Hybrid polysaccharide-silica nanocomposites prepared by the sol-gel technique. Langmuir 20 3882-3887 (2004)

8. Shchipunov, Y.A.: Entrapment of biopolymers into sol-gel-derived silica nanocomposites. In: Ruiz-Hitzky, E., Ariga, K., Lvov, Y. (eds.) Bio-Inorganic Hybrid Nanomaterials, pp. 75-117. Wiley-VCH Verlag, Weinheim (2008)

9. Li, H., Jo, J.K., Zhang, L., Ha, C.S., Suh, H., Kim, I.: A general and efficient route to fabricate carbon nanotube-metal nanoparticles and carbon nanotubeinorganic oxides hybrids. Adv. Func. Mater. 20, 3864-3873 (2010)

10. Sutherland, R.L.: Handbook of Nonlinear Optics. Marcel Dekker, New York (2003)

11. Agrawal, G.P.: Nonlinear Fiber Optics. Academic, San Diego (2001)

12. Stentz, A.J., Boyd, R.W.: Nonlinear optics. In: Gupta, M.C., Ballato, J. (eds.) The Handbook of Photonics, pp. 5-1-5-26. CRC Press, Boca Raton (2007)

13. Sanches, C., Ribot, F., Lebeau, B.: Molecular design of hybrid organicinorganic nanocomposites synthesized via sol-gel chemistry. J. Mater. Chem. 9, 35-44 (1999)

14. Palpant, B.: Third-order nonlinear optical response of metal nanoparticles. In: Papadopoulos, M.G., Sadlej, A.J., Leszczynski, J. (eds.) Non-Linear Optical Properties of Matter. From Molecules to Condensed Phases, pp. 461-508. Springer, Dordrecht (2006)

15. Innocenzi, P., Lebeau, B.: Organic-inorganic hybrid materials for non-linear optics. J. Mater. Chem. 15, 3821-3831 (2005)

doi:10.1186/2228-5326-2-13

Cite this article as: Postnova et al:: Tailored hybrid hyperbranched polyglycidol-silica nanocomposites with high third-order nonlinearity. International Nano Letters 2012 2:13. 